Assurances et gestion des risques

Insurance and Risk Management

\title{
Les déterminants et l'effet du management des risques sur la performance dans les entreprises non financières : un état de l'art
}

\section{Kouakou Armel Assienin}

Volume 86, numéro 1-2, juin 2019

URI : https://id.erudit.org/iderudit/1062462ar

DOI : https://doi.org/10.7202/1062462ar

Aller au sommaire du numéro

\section{Éditeur(s)}

Faculté des sciences de l'administration, Université Laval

\section{ISSN}

1705-7299 (imprimé)

2371-4913 (numérique)

\section{Découvrir la revue}

Citer cet article

Assienin, K. A. (2019). Les déterminants et l'effet du management des risques sur la performance dans les entreprises non financières : un état de l'art. Assurances et gestion des risques / Insurance and Risk Management, 86(1-2), 1-30. https://doi.org/10.7202/1062462ar
Résumé de l'article

Cet article a pour objectif de faire une synthèse de la littérature pour les managers, les chercheurs et les praticiens sur les fondements, les déterminants et les résultats de la mise en oeuvre d'une politique de management des risques sur la performance. Pour atteindre cet objectif, l'article est subdivisé en trois parties. La première présente les fondements de la gestion des risques dans le contexte de la théorie des firmes, la deuxième donne les principaux déterminants du management du risque et, pour terminer, la troisième fait un état de l'art des études empiriques sur l'influence du management du risque sur la performance des entreprises non financières.
Tous droits réservés $\odot$ Faculté des sciences de l'administration, Université Laval, 2019
Ce document est protégé par la loi sur le droit d'auteur. L'utilisation des services d'Érudit (y compris la reproduction) est assujettie à sa politique d'utilisation que vous pouvez consulter en ligne.

https://apropos.erudit.org/fr/usagers/politique-dutilisation/ 


\section{LES DÉTERMINANTS ET L'EFFET DU MANAGEMENT DES RISQUES SUR LA PERFORMANCE DANS LES ENTREPRISES NON FINANCIÈRES: UN ÉTAT DE L'ART}

Kouakou Armel ASSIENIN ${ }^{1}$

\section{RÉSUMÉ}

Cet article a pour objectif de faire une synthèse de la littérature pour les managers, les chercheurs et les praticiens sur les fondements, les déterminants et les résultats de la mise en ouvre d'une politique de management des risques sur la performance. Pour atteindre cet objectif, l'article est subdivisé en trois parties. La première présente les fondements de la gestion des risques dans le contexte de la théorie des firmes, la deuxième donne les principaux déterminants du management du risque et, pour terminer, la troisième fait un état de l'art des études empiriques sur l'influence du management du risque sur la performance des entreprises non financières.

Mots clés : Management du risque - Performance - Performance globale

\section{ABSTRACT}

The aim of this article is to summarize the literature for managers, researchers and practitioners on the foundations, determinants and results of the implementation of a risk management policy on performance. To achieve this goal, the article is subdivided into three parts. The first presents the fundamentals of risk management in the context of firm theory, the second provides the main determinants of risk management, and the third concludes with a state-of-the-art of empirical studies on risk management and its influence on the performance of non-financial companies.

Keyword : Risk management - Overall performance and performance 


\section{INTRODUCTION}

Dans le contexte actuel des affaires, les entreprises sont de plus en plus confrontées à une forte concurrence, ce qui les met dans l'obligation de prendre des risques pour se positionner ou se démarquer de leurs concurrents, et cela, en investissant dans de nouveaux projets avec lesquels le personnel n'est pas toujours familier. Il peut s'agir d'innovation, d'entrée sur de nouveaux marchés ou de révision de la stratégie de production. Peu importe la nature des investissements, plus le degré de nouveauté sera grand par rapport aux compétences et à l'expérience de l'entreprise, plus ceux-ci seront empreints d'incertitude. L'histoire sur les causes de faillite liées à la négligence des risques a montré que la mise en œuvre d'une politique de gestion des risques pouvait faire toute la différence entre les entreprises en faillite et les entreprises en bonne santé financière.

En outre, il convient de rappeler la distinction faite dans la littérature au sujet du risque systémique et du risque spécifique. En effet, l'entreprise peut être confrontée à des chocs potentiellement dangereux et hors du contrôle de leurs gestionnaires qui peuvent affecter le système économique dans son ensemble (Bordes, 1999). On se retrouve donc dans le cas de risque systémique. Est-il possible de gérer ce type de risque? Qui doit gérer ce type de risque? Nous pensons comme Bordes (1999) que seuls les pouvoirs publics en sont capables car ils ne peuvent pas être gérés par l'entreprise (Vernimmen, 2012). Pour ce qui nous concerne, dans ce travail, nous nous focaliserons sur le risque spécifique qui pour nous peut être géré et couvert par la politique mise en place par l'entreprise. Il est la conséquence d'une mauvaise gestion, des évènements économiques ou financiers (Vernimmen, 2012).

Dans cette perspective, il nous parait intéressant d'analyser jusqu'à quel point l'utilisation d'une politique de couverture des risques au sein d'une entreprise pouvait déterminer la performance financière de celle-ci. Ce sujet d'actualité paraît tellement important que Karim et Souad (2010) ont souligné que "la gestion des risques par les entreprises non-financières est considérée comme un des thèmes les plus importants par les chercheurs en gestion et plus particulièrement en finance d'entreprise".

Par ailleurs, comme Razali et Tahir (2011), nous pensons que les travaux sur le management des risques restent encore limités. C'est ce qui motive ce travail de recension des écrits en vue de mettre à disposition des managers de gestion des entreprises non financières et des chercheurs une synthèse des écrits leur permettant de relever les 
fondements de la gestion des risques dans le contexte des théories de la firme et d'identifier les pratiques de gestion des risques ayant un impact sur la performance des entreprises.

Toutefois, nous solliciterons les théories de la firme (parfois même des théories concurrentes), pour justifier la diversité des raisons qui fondent l'utilisation des politiques de gestion de risque dans l'entreprise.

Dans la réflexion qui suit, nous présenterons les bases théoriques de la gestion des risques, les travaux empiriques et théoriques menés sur l'influence de l'adoption d'une politique de couverture sur la performance.

\section{FONDEMENTS THÉORIQUES DE LA GESTION DES RISQUES}

\subsection{La théorie des droits de propriété}

Selon Pejovich (1978), "[...] les droits de propriété ne sont pas des relations entre les hommes mais des relations entre les hommes qui ont rapport à l'usage des choses [...]" Pour lui, il y a une relation entre la dimension de la firme, l'intensité de la concurrence et le contenu des droits de propriété. L'atténuation des droits du propriétaire s'explique aussi par la dimension de la firme et par la structure du marché sur lequel elle évolue.

Charreaux et Pitol-Belin (1992) estiment qu'un droit de propriété n'est complet que s'il remplit les conditions d'exclusivité et de transférabilité. L'exclusivité implique une liberté entière dans l'utilisation des actifs possédés ainsi que leur contrôle total et la transférabilité se traduit par une possibilité d'échange qui ne dépend que de l'acheteur et du vendeur. Car l'économie du marché est fondée sur la liberté des échanges et respecte pleinement les droits de propriété. Elle permet donc à chaque agent de maximiser son utilité et elle représente un système économique optimal. Mais la réalité est toute autre; en effet, plusieurs facteurs, tels que les lois et les coûts de transaction, atténuent le droit de propriété.

Cette théorie a par ailleurs déclenché plusieurs études sur la comparaison de l'efficacité des systèmes des organisations. C'est le cas des études de Furubotn et Pejovich (1972), de Simon et al. (1977) et de 
Desbrières (1990) qui comparent l'efficacité des formes organisationnelles suivantes: la firme réglementée, la firme managériale, les mutuelles et les coopératives, la firme publique, la firme socialiste et la firme autogérée (Charreaux et Pitol-Belin, 1992). Les apports de cette théorie peuvent également s'apprécier au niveau des innovations dans les problèmes soulevés à cette époque. Pour Tinel (2004), cette théorie a soulevé de nouveaux problèmes (à cette époque) tels que l'aléa moral, la mesure de la productivité individuelle et le contrôle, à partir desquelles vont se développer la théorie de la firme et des organisations, la théorie des contrats, etcetera.

Malgré ces apports, cette théorie a montré ses limites dans l'explication de la structure et du fonctionnement des organisations car l'essentiel de l'analyse porte sur les rapports entre propriétaires et dirigeants (Charreaux et Pitol-Belin, 1992). Tinel (2004) estime quant à lui que le cadre d'analyse de la théorie des droits de propriété est trop restreint. Pour lui, c'est l'une des raisons pour laquelle le modèle d'Alchian et Demsetz (1972) n'a pas servi de cadre de référence aux approches ultérieures en matière de théorie de la firme.

L'utilisation de cette théorie comme fondement de la gestion des risques se justifie par les propos de Demsetz (1967). Selon ce dernier "les droits de propriété permettent aux individus de savoir à priori ce qu'ils peuvent raisonnablement espérer obtenir dans leurs rapports avec les autres membres de la communauté. Ces anticipations se matérialisent par les lois, les coutumes et les mours d'une société. Détenir des droits, c'est avoir l'accord des autres membres de la communauté pour agir d'une certaine manière et attendre de la société qu'elle interdise à autrui d'interférer avec ses propres activités, à la condition qu'elles ne soient pas prohibées". Ainsi donc, cette capacité d'anticipation que nous confère la théorie des droits de propriété nous conduit à la considérer comme un fondement de la gestion des risques. Car elle emmène les entreprises à adopter des pratiques de couverture des risques telles que les contrats de travail, de prestation etcetera.

\subsection{La théorie des droits de l'agence}

La théorie de l'agence vient en complément ou vient généraliser la théorie des droits de propriété (Charreaux et Pitol-Belin, 1992). Jensen et Meckling (1976) définissent la relation d'agence "comme un contrat par lequel une ou plusieurs personnes (le principal) engagent une autre personne (l'agent) pour exécuter en son nom une tâche quelconque qui implique une délégation d'un certain pouvoir de décision à l'agent ». La relation d'agence s'applique même à de simples 
coopérations telles que le propriétaire d'un véhicule qui va voir un mécanicien, le malade qui paie le médecin pour des soins de santé ou le gestionnaire qui embauche un ingénieur pour un travail dans l'entreprise. Cette théorie met en évidence un problème d'information dans la mesure où le mandant ne dispose pas des mêmes informations que le mandataire. Cette asymétrie d'informations emmène les individus à maximiser leur fonction d'utilité ce qui entraine des intérêts divergents.

Les divergences d'intérêts entre les propriétaires et les dirigeants, la difficulté pour les propriétaires de contrôler parfaitement les efforts des dirigeants pour accomplir leurs tâches, les conduisent à investir dans la surveillance, le contrôle direct de l'action des agents et dans la gestion des risques. Ces coûts supplémentaires qu'ils supportent sont appelés "coût d'agence». Par conséquence, la théorie de l'agence constitue un fondement de la gestion des risques dans les entreprises. Pour l'appliquer à notre sujet, nous considérons les actionnaires des entreprises (principal) dans leur relation avec le dirigeant (l'agent). Ce dernier dans sa relation avec les employés, nous remarquons que l'enjeu est de créer les circonstances pour contraindre l'agent à se conformer au contrat de travail qui les lient. Pour cela, le principal est tenu de prendre des mesures pour se prévenir du risque que l'agent travaille pour son propre intérêt au détriment de celui du principal.

\subsubsection{Risque moral}

Le risque moral c'est le risque qu'encourt le principal du fait que l'agent peut tirer profit de l'asymétrie d'informations pour accroître sa satisfaction. Ce risque est dû par le fait que le principal ne dispose pas de toute l'information permettant d'évaluer l'agent et savoir s'il travaille pour satisfaire ses propres intérêts ou ceux du principal. Taylor (1929), disait qu'«il n'est pas rare qu'un ouvrier compétent découvre en peu de temps comment travailler moins tout en donnant l'impression à son employeur qu'il travaille beaucoup". Certes, l'une des causes de ce risque est que l'agent n'est pas toujours rémunéré en fonction de sa contribution dans sa création de la richesse, mais il n'en demeure pas moins que plusieurs personnes, malgré qu'elles soient bien rémunérées, cherchent leur intérêt au détriment de celui du principal. C'est tout l'intérêt des pratiques de gestion de risque qui, une fois adoptées et mises correctement en œuvre, permettent de limiter le risque moral et cela à travers un système qui incite l'agent à travailler dans l'intérêt du principal (Jost, 2004). Ces pratiques de gestion prennent différentes formes. Ce sont: les contrats, les pratiques de gestion, les assurances, etcetera. 
Le principal ne disposant pas de toutes les informations, il est pratiquement impossible d'avoir des contrats qui puissent prévoir tous les risques qui peuvent survenir, ni toutes les conséquences de l'exécution des contrats établis. C'est ce qui explique l'insuffisance des contrats dans la plupart des relations d'agence.

Comme nous l'avons dit plus haut, la théorie de l'agence met en évidence deux types d'individu: ceux qui cherchent à maximiser leur fonction d'utilité (l'agent) et ceux qui cherchent à anticiper l'impact de cette relation d'agence sur leur propriété (le principal). En conséquence dans ce partenariat, l'agent cherche à profiter des failles ou de l'incomplétude des contrats. Cette attitude conduira le principal à investir dans le contrôle direct de l'agent, d'où les coûts de surveillance. Ces coûts peuvent apparaître sous forme de sollicitation de cabinet d'audit en vue d'auditer les entreprises pour déceler les pratiques visant à maximiser la fonction d'utilité de l'agent au détriment de celui du principal. Ce contrat est donc avant tout une attestation de confiance entre le Principal et l'Agent où les situations réciproques sont connues des deux acteurs, étant entendu qu'une situation initiale de confiance limite les coûts de transaction dits ex-post (comme par exemple des coûts de transaction résultant de l'apparition d'un litige dans la réalisation du contrat).

Cela dit, pour les raisons exposées plus haut, l'information est rarement parfaite comme le suppose l'idéal type du marché walrasien. Cela a été montré plus haut dans ce travail: dans l'hypothèse d'une parfaite information entre les acteurs et de l'attribution de droits de propriété négociables, la résolution des externalités peut se faire par le biais du marché, donc sans avoir besoin d'une intervention de l'autorité. Mais ce serait négliger la question de coûts de transaction (supposés positifs - théorème de Coase) et le problème d'opportunisme des acteurs. Oliver E. Williamson (1975), dans un ouvrage qui a fait sa renommée et que nous traiterons dans le chapitre suivant, explique très bien cela.

Partant du postulat de rationalité limitée des acteurs lors de la conclusion du contrat, Williamson (1975) affirme que le résultat final est souvent l'incomplétude matérielle du contrat, situation laissant des zones d'ombre dans l'application du contrat (les parties ne pouvant prévoir à l'avance toutes les éventualités résultant de l'application du contrat), permettant des comportements opportunistes des acteurs, dont la résultante peut prendre les deux formes suivantes: 
1) L'aléa moral (moral hazard), appelé aussi risque moral. Il s'agit de la forme ex-post d'opportunisme (ou opportunisme post contractuel). Il se présente dans la phase d'exécution du contrat lorsque l'incomplétude du contrat est telle qu'il y a une réelle difficulté à déterminer si une partie a respecté son engagement ou non. On dit alors que dans cette éventualité, l'autre partie supporte un risque moral de la correcte exécution du contrat par son co-contractant.

2) La sélection adverse, appelé aussi anti-sélection, fait allusion à une forme ex-ante d'opportunisme. En effet, dans ce cas de figure, il y a triche avant la passation d'un contrat (par exemple, dans la fourniture de faux renseignements de l'une des parties sur l'objet de la transaction).

Pour ce qui nous concerne, nous allons nous pencher dans le point suivant sur la théorie des contrats de Williamson (1975), intégrant mieux que quiconque dans sa typologie des contrats les variables de rationalité limitée et d'opportunisme, les deux variables centrales du risque moral.

\subsection{La théorie des coûts de transaction}

La théorie des coûts de transaction a été construite au fil des années par les principaux auteurs qui sont Coase et Williamson. Elle a valu à son auteur Coase (1937), qui en est le précurseur, un prix Nobel en 1991. Toutefois, c'est avec les travaux de Williamson (1975) que la théorie de transaction prendra toute sa place. En effet, ses travaux ont rendu plus opérationnelle la théorie et ont permis d'analyser les transactions entre le marché et la hiérarchie en prenant en compte la rationalité limitée.

La question fondamentale à laquelle Coase (1937) essaie de répondre est: pourquoi existe-t-il des firmes dans une économie de marché? Dans son article fondateur, Coase (1937) répond à la question en présentant les deux formes de coordination économique existantes. Il s'agit de la coordination sur le marché par le système des prix et de la coordination administrative par la hiérarchie au sein de l'entreprise. Il poursuit en disant qu'une firme apparaît quand la passation d'un contrat à très court terme n'est pas satisfaisante. Une entreprise tendra à s'agrandir jusqu'à ce que les coûts de transaction supplémentaires en son sein deviennent égaux au coût de réalisation de cette même transaction par le biais d'un échange sur le marché ou aux coûts 
d'organisation dans une autre entreprise. Pour Williamson (1994) l'économie des coûts de transaction, couvre un champ relativement large et possède une application assez étendue. Selon lui, toute relation économique ou autre prend la forme d'un problème contractuel ou peut être décrit comme tel. Il peut par conséquent être évalué avantageusement selon les termes de l'économie des coûts de transaction. Abecassis (1997), quant à elle, pense que cette théorie tente de définir ce que sont les notions de marché et de hiérarchie (synonyme d'entreprise ou d'organisation), en justifiant leur existence simultanée. En effet, les économistes classiques et néoclassiques partent de l'hypothèse selon laquelle le marché est le mode de coordination optimal, ce qui pose la question de la justification de l'existence de l'entreprise.

Ainsi donc, la théorie des coûts de transaction fournit une grille d'analyse qui permet de répondre à de nombreuses questions parmi lesquelles l'évaluation des impacts organisationnels des nouvelles technologies de l'information et de la communication, mais aussi au choix micro-économique de faire ou faire faire. Elle s'avère également très utile pour expliquer le mouvement d'externalisation de certaines fonctions, encore appelé "outsourcing". Cette grille d'analyse peut aussi permettre d'expliquer des choix organisationnels, au niveau de firmes ou de filières entières. Williamson (1975) dira pour sa part que le recours à la hiérarchie est surtout développé là où les transactions sont récurrentes, exécutées dans un climat d'incertitude et qui nécessitent des investissements idiosyncrasiques (très spécifiques). Donc au fur et à mesure que l'incertitude augmentera, les transactions récurrentes vont être soit standardisées et confiées au marché, soit internalisées.

On déduit donc que la théorie des coûts de transaction même si elle connaît des difficultés d'opérationnalisation, constitue un fondement de la gestion des risques. En effet, elle permet de faire un arbitrage entre l'internalisation et l'externalisation. Williamson (1975), en s'appuyant sur deux hypothèses comportementales que sont l'opportunisme et la rationalité limitée, montre que le risque d'opportunisme augmente les coûts de transaction, c'est-à-dire le coût de négociation et de supervision d'un contrat. Donc l'existence d'opportunisme incite à l'internalisation de la transaction. En effet, le recours à l'entreprise se justifie pour se protéger contre le risque d'opportunisme. Toutefois, en se focalisant sur l'hypothèse de la rationalité parfaite d'Herbert Simon (1978), on pourrait justifier l'adoption des pratiques de couverture de risque. 


\subsection{La théorie de la rationalité limitée}

Les travaux d'Herbert Simon, prix Nobel d'économie en 1978, s'inspirent de la théorie économique standard. En effet, les modèles classiques supposent que les individus sont rationnels, avec l'hypothèse que les hommes obéissent à une rationalité parfaite. Construit sur une base idéaliste et abstraite, ce modèle a fait l'objet de plusieurs critiques. Friedman (1953) dira à ce sujet que "tout se passe comme si les comportements individuels étaient régis par une rationalité parfaite; il n'est pas nécessaire que cette hypothèse soit réaliste, il suffit que les prédictions du modèle construit sur la base de cette hypothèse soient conformes à la réalité».

\subsubsection{De l'hypothèse de la rationalité parfaite à l'hypothèse de la rationalité limitée.}

\subsubsection{L'hypothèse de la rationalité parfaite}

Cette hypothèse constitue l'un des fondements de la science économique, surtout celle de la microéconomie. Car elle présente la procédure d'arbitrage entre les différentes options possibles qui s'offrent à l'homme rationnel. Elle constitue une sorte de norme et établit le lien nécessaire entre la raison et l'action (Popper, 1963). En se basant sur cette hypothèse, les modèles des économistes auraient un caractère arbitraire qui deviendrait esprit de caprice (Popper, 1963). L'homo economicus y est présenté comme un personnage qui ne retient que son intérêt personnel dans ses prises de décision, qui ne cherche qu'à maximiser son utilité. Il dispose de toutes les capacités cognitives et d'informations parfaites pour d'une part construire des critères de choix entre les différentes actions possibles, d'autre part déterminer le choix qui satisfait au mieux ces critères en respectant ces contraintes. La théorie de la rationalité parfaite a certes permis des avancées notables dans l'analyse économique mais elle a aussi fait l'objet de plusieurs critiques. Ce qui a permis de considérer d'autres conceptions de la rationalité des comportements.

Ce qui nous conduit aux travaux de Simon (1955), précurseur de la théorie de la rationalité limitée basé sur l'irréaliste de la rationalité parfaite ont montré que d'autres conceptions de la rationalité des comportements pouvaient donner une image beaucoup plus fidèle et réaliste des comportements individuels. Malgré toutes les assurances données par des chercheurs tels que Friedman (1953), le caractère abstrait et irréaliste du modèle classique fera l'objet de plusieurs critiques qui nous feront passer de la rationalité parfaite à la rationalité limitée. 


\subsubsection{L'hypothèse de la rationalité limitée}

Le succès de cette hypothèse s'observe dans la littérature à travers les travaux de plusieurs économistes. C'est le cas de Laville (1998) avec ses tentatives de modélisation de la "rationalité limitée", de Walliser (1989) avec ses extensions de "rationalité cognitive", de Ponssard (1994) avec ses travaux sur la "rationalité interactive", de Orlean (1994) avec ses travaux sur "la rationalité située» (Cozic, 2018).

Selon Béjean et al. (1999), toutes les interprétations de la rationalité limitée sont très diverses et ne sont pas toujours fidèles à la pensée de Herbert Simon. Toutefois, ces interprétations de la rationalité simonienne se rattachent implicitement à des positions méthodologiques différentes. Leurs recherches ont permis de répartir les interprétations de l'hypothèse simonienne en deux approches différentes de la rationalité limitée:

- La première fonde l'hypothèse de la rationalité limitée sur les limites des capacités cognitives des individus et la conçoit comme une simple atténuation de la rationalité parfaite.

- La deuxième met non seulement les capacités cognitives limitées des individus en évidence mais aussi sur l'instabilité des états de la nature (l'ensemble des possibilités d'action et de leurs conséquences associés). Elle s'attache, selon Béjean et al. (1999), non plus à la décision mais au processus de décision. En conséquence, apparaît une autre hypothèse de rationalité individuelle qui ne résulte pas d'une simple limitation de la rationalité parfaite.

Pendant que l'hypothèse de la rationalité parfaite présente l'homme rationnel comme un être ayant connaissance de la liste prédéterminée des états de la nature, au point d'avoir une connaissance parfaite des évènements futurs, avec des capacités cognitives et des capacités d'acquisition d'informations qui lui permettent de se faire une image fidèle de la réalité; l'hypothèse de rationalité limitée met en avant l'irréalisme de la théorie de la rationalité parfaite. Elle commence par lever l'hypothèse de la parfaite connaissance des évènements futurs et à présenter les limites de l'homme quant à ses capacités cognitives et ses capacités d'acquisition d'information. En effet, au moment de sa prise de décision, l'individu ne possède qu'un fragment de l'information disponible sur l'avenir (Béjean et al., 1999). Viviani (1994) dira pour sa part que le paysage existe déjà, mais il est souvent si complexe qu'il est toujours possible de découvrir de nouveaux sentiers. Cela pour dire que l'individu découvre au fil du temps les opportunités qui ne sont pas encore exploitées. 
A partir des travaux de Simon (1955), on se rend compte que les partisans de la rationalité parfaite surestiment les capacités cognitives de l'individu. En considérant les limites de ces capacités, on réalise que l'individu ne peut avoir une parfaite perception des évènements futurs. Elles réduisent la capacité à s'informer et à utiliser toutes les informations disponibles. Chanut et al. (2011) estiment que les limites des capacités cognitives et physiques ont des conséquences sur les possibilités physiques et intellectuelles des individus.

Par ailleurs face à la complexité et à l'incertitude du monde présent, les limites des capacités de l'individu l'obligent à adopter les pratiques de couvertures de risques. L'hypothèse de la rationalité limitée a permis de mettre en évidence les limites de l'individu qui, dans un monde aussi complexe, ne peut disposer d'informations sur toutes les possibilités qui s'offrent à lui et sur toutes les conséquences de ses actions. Ainsi pour l'entrepreneur qui ne dispose pas de toutes les informations sur les stratégies de ses concurrents, sur le marché, sur la demande et sur les catastrophes naturelles, se voit dans l'obligation de souscrire de l'assurance, à élaborer des contrats commerciaux, en vue de prévenir des risques de pertes énormes qui pourraient avoir des conséquences tragiques sur son entreprise. Nous déduisons donc que la théorie de la rationalité limitée est un fondement de l'adoption des pratiques de couverture de risque.

\subsection{La théorie administrative}

Fayol (1962), l'un des précurseurs de l'école classique du management, s'est intéressé dans ses travaux à la direction de l'entreprise. C'est ce qui l'a conduit à jeter les bases de la théorie administrative. Selon Fayol (1962), les activités d'une entreprise peuvent se présenter sous 6 fonctions qui sont: la fonction technique, la fonction commerciale, la fonction financière, la fonction comptable, la fonction de sécurité qui consiste à assurer la protection des personnes et des biens et la fonction administrative.

Ainsi, Fayol, déjà en 1962, avait vu l'importance de la gestion des risques qui à ce moment consistait à assurer la sécurité des personnes et des biens. Ainsi donc une bonne gestion administrative doit tenir compte des risques. Car les actionnaires ont besoin d'assurer la sécurité de leur investissement et du personnel qui travaille pour eux. La théorie administrative apparaît donc comme un fondement de l'adoption des pratiques de couverture des risques. 


\subsection{La théorie de la hiérarchisation des besoins}

Maslow (1954), spécialiste de la personnalité, dans la poursuite des travaux de Mayo (1949) s'est accentué sur les besoins de l'homme afin de mieux comprendre ce que ce dernier recherche dans son travail. Son hypothèse de base est que le comportement de l'homme dans son service sera plus productif s'il y trouve la satisfaction de ses besoins. Ainsi, il proposera une hiérarchisation des besoins qui est jusqu'aujourd'hui diffusée dans les domaines de la gestion des ressources humaines et du marketing comme un outil pour cerner le comportement des employés et des consommateurs. Sa pyramide comporte cinq niveaux et chaque niveau exprime un besoin qui motive l'homme dans son travail. Il s'agit du besoin physiologique, besoin de sécurité, besoin d'appartenance, besoin de reconnaissance et du besoin d'accomplissement. Le besoin de sécurité qui nous intéresse dans le cadre de notre étude prend en compte le besoin de sécurité physique et économique.

Nous pensons que l'entreprise, en tant que personne morale tout comme le propriétaire, a aussi le besoin de sécurité dans la mesure où dans un environnement instable l'entreprise ne peut pas prospérer. Ce besoin de sécurité peut expliquer l'aversion au risque des managers ou des actionnaires. Elle est sans doute l'une des raisons qui justifie les pratiques de couverture du risque.

\section{LES DÉTERMINANTS DU MANAGEMENT DU RISQUE}

L'introduction des frictions de marché par le modèle Modigliani et Miller (1958), en ce qui concerne la structure optimale du capital, a suscité le débat sur les déterminants de la couverture des risques. Ainsi plusieurs recherches empiriques ont été menées sur le sujet.

Froot, Scharfstein et Stein (1993) ont présenté un cadre d'analyse du management des risques et de la rationalité financière. Pour eux, le recours à des pratiques de gestion des risques, du fait que les imperfections du marché financier rendent les coûts des fonds extérieurs plus élevés que ceux obtenus intérieurement, est rationnel. Aretz, Bartram et Dufey (2007) diront quant à eux que la couverture répond à l'aversion au risque du gestionnaire et permet aussi de régler les problèmes de substitution d'actif en réduisant la volatilité des cash flows à travers une réduction du risque non systématique. Raïs (2012) dira, pour ce qui le concerne, qu'en réduisant d'une part les financements externes liés aux dépenses de faillite et aux impôts (réduire les 
fluctuations dans le revenu avant impôts) et d'autre part les dépenses associées aux conflits d'agence, le management des risques pourra augmenter la valeur de l'entreprise. La présente section s'articule autour des trois catégories de couverture de risques qui sont présentées dans la littérature.

\subsection{Les déterminants fondés sur l'hypothèse de la maximisation de valeur de l'entreprise}

La couverture du risque peut avoir pour objectif la maximisation de la valeur de l'entreprise. Ainsi, la stratégie de gestion des risques utilisée permet de réduire les impôts à payer et les coûts de la détresse financière. Par ailleurs, elle permet de garantir ou d'augmenter le niveau de liquidité de l'entreprise.

\subsubsection{La réduction de l'impôt à payer}

Les travaux de Smith et Stulz (1985) et Stulz (1996) ont montré que la couverture du revenu avant impôt pouvait augmenter la valeur de l'entreprise à condition que sa structure d'impôt soit convexe. Car lorsque l'entreprise rencontre un taux d'imposition marginal progressif impliquant une fonction d'impôt convexe, la gestion des risques peut donc réduire le montant d'impôt attendu en réduisant la volatilité du revenu avant impôt. Mefteh (2005), dans son étude, a démontré à partir de l'exemple d'une entreprise française confrontée au risque de change que la couverture du risque permet non seulement de stabiliser son revenu mais aussi d'accroître son bénéfice net d'impôt moyen. Cela est dû, selon elle, aux différences dans le traitement fiscal des gains et des pertes. Car les reports des pertes sur les exercices ultérieurs et les crédits d'impôts expliquent les gains d'impôts et sont à la base de la convexité de la fonction d'imposition. Ce point de vue de Mefteh (2005) vient donc confirmer les propos de Graham et Smith (1999). Pour ces derniers, une entreprise a une plus grande probabilité d'avoir une fonction d'impôt convexe lorsque son revenu imposable est proche de zéro et est aléatoire ou encore lorsqu'il existe une corrélation négative entre les revenus des différentes périodes.

\subsubsection{La réduction des coûts de faillites}

Une entreprise qui ne peut pas honorer ses engagements financiers à l'échéance prévue se trouve en situation de défaillance. Une telle situation entraîne des coûts financiers qui peuvent être soit des coûts 
directs, tels que les coûts légaux et administratifs, soit des coûts indirects associés, par exemple, à la détérioration des relations avec les partenaires de l'entreprise (Mellios, 2001). Les coûts de faillite constituent une des raisons qui motivent les entreprises dans l'adoption de politiques de couverture de risque. Mayer et Smith (1982) puis Smith et Stulz (1985) ont montré que la couverture de risque (n'entrainant pas de coût) était un moyen qui permettait de réduire cette probabilité en diminuant la variance de la valeur firme et donc de réduire les coûts de faillite attendus. Robichek et Myers (1966) considèrent que les coûts de faillite sont supportés par l'entreprise dès qu'un risque de défaillance est constaté et ce, même si les difficultés financières sont surmontées par la suite. Brealey et Myers (1984) attestent que les coûts de faillite sont importants et significatifs et qu'il convient d'en tenir compte lors de la prise de décisions financières à long terme et à court terme. Les pratiques de gestion des risques permettent de contrer les coûts de faillite à travers la valeur qu'elle crée pour l'entreprise et en diminuant les occasions de devoir faire face à des difficultés financières. Car par la réduction des décalages entre les entrées et les sorties de fonds, elles minimisent les probabilités qui conduiraient l'entreprise à se trouver en cessation de paiement (Mefteh, 2005).

Ainsi, la réduction des coûts de faillite est une raison suffisante pour adopter les pratiques de couverture de risque. Smith et Stulz (1985) diront, pour ce qui les concerne, que la réduction des coûts de faillite est un motif déterminant de la couverture des risques. Selon eux, en cas d'endettement, l'augmentation de la valeur de l'entreprise accroît la probabilité de remboursement des créanciers et la valeur de la part résiduelle revenant aux actionnaires. Comme la couverture diminue la variabilité de la valeur future de l'entreprise, la probabilité d'encourir un risque de faillite se trouve diminuée, ce qui devient avantageux aussi bien pour les créanciers que pour les actionnaires. Par ailleurs, ils montrent que la couverture améliore la réputation de l'entreprise qui emprunte fréquemment sur le marché financier et augmente ainsi le prix des obligations émises.

\subsubsection{La réduction des coûts de sous-investissement}

La volatilité des revenus peut avoir un impact négatif sur l'investissement parce que, d'un côté, elle contraint l'entreprise à laisser passer des opportunités d'investissement rentables et, d'un autre, elle la contraint à chercher des ressources externes dans les périodes de faible rentabilité (Mefteh, 2005). Mian (1996) dira quant à lui que les coûts de financement externes comportent des coûts indirects comme les 
coûts d'agence de la dette. Pour lui ces coûts entrainent un sous-investissement si l'entreprise n'a pas une capacité d'autofinancement. Les investissements des entreprises sont fonction des flux monétaires dégagés. Car elles sont dans l'obligation de planifier leurs investissements surtout lorsque les flux monétaires sont très aléatoires. La capacité qu'a la couverture des risques de réduire la variabilité des flux monétaires peut permettre d'augmenter la valeur de l'entreprise en évitant des financements externes couteux et permettra de saisir toute opportunité d'investissement. Pour Bessembinder (1991), la couverture permet de diminuer les incitations à sous-investir. Ce dernier, à travers son analyse, montre qu'une augmentation du nombre de contrats à terme diminue au minimum le coût du sous-investissement. L'étude de Smith et Watts (1992) s'est focalisée sur la relation entre la couverture et l'endettement. Ils montrent à travers leur étude la difficulté de séparer deux effets inverses de l'endettement sur les décisions de couverture.

- Effet 1: si l'entreprise dispose d'opportunités d'investissement, un niveau d'endettement élevé entrainera la couverture des risques.

- Effet 2 : les entreprises endettées ont généralement peu d'options de croissance et sont moins motivées à couvrir leurs risques.

De ce fait, les entreprises disposant de plusieurs opportunités d'investissement sont incitées à baisser leur niveau d'endettement, à raccourcir la maturité des dettes, à payer moins de dividendes et à augmenter le niveau de liquidité. Elles ont besoin de couvrir leurs risques. Ces auteurs estiment que la stratégie optimale de la couverture, en termes de montant de la couverture et des instruments utilisés, dépend de l'interaction entre les opportunités d'investissement et de financement. Ils préconisent qu'un programme de gestion de risque bien conçu peut permettre à l'entreprise de coordonner ses politiques d'investissement et de financement. A partir des travaux des chercheurs cités plus haut, nous déduisons que l'adoption des pratiques de gestion des risques est un moyen de résolution des problèmes de sous-investissement liés à l'insuffisance des richesses générées par l'entreprise.

\subsubsection{Les instruments du bilan comme moyen de couverture}

Contrairement à plusieurs auteurs qui pensent que la volatilité des rendements de l'entreprise peut se contrôler à travers les produits dérivés, Nance et al. (1993) proposent l'utilisation des instruments de gestion ou du bilan. A titre d'exemple, pour une entreprise qui est confrontée à un risque de taux d'intérêt, elle peut agir sur la structure de capital ou fixer le ratio de la dette à son minimum. Leur étude 
montre que la liquidité est un substitut de la couverture. Ainsi, les entreprises disposant de liquidité ou de titres (actions, obligations convertibles en actions) ont plus de capacité à faire face aux échéances ou d'éviter de tomber en défaut de paiement.

\subsection{Les déterminants fondés sur l'hypothèse de la maximisation de la richesse ou de l'utilité des managers de l'entreprise}

L'hypothèse de la maximisation des managers de l'entreprise trouve son fondement dans la théorie de l'agence développé par Jensen et Meckling (1976). En effet, pour ces chercheurs la relation d'agence est un contrat qui lie une ou plusieurs personnes (le principal) à une autre personne (l'agent) pour effectuer ou accomplir une tâche ou un service qui nécessite la délégation d'une partie de l'autorité à l'agent. La relation d'agence, telle que définie, est source de conflits à cause de la divergence d'intérêt des parties prenantes. Les décisions prises par l'agent ne sont pas toujours dans l'intérêt du principal. La résolution de ces conflits d'intérêt provoque des coûts (Jensen et Meckling, 1976). Ce sont:

- Les coûts de surveillance ou de contrôle: frais engagés par le principal pour tenter de limiter le comportement opportuniste de l'agent.

- Les coûts d'obligation: frais engagés par l'agent pour convaincre le principal qu'il œuvre dans ses intérêts.

- Les coûts résiduels: coûts correspondant à la perte d'utilité subie par l'une ou l'autre des parties à la suite de la divergence des intérêts.

Pour Jensen et Meckling (1976) les agents sont supposés chercher leur propre intérêt au dépend de celui du principal. Ce comportement, qu'ils qualifient d'opportuniste, se voit dans le choix des décisions de couverture des risques financiers de l'entreprise. Cliche (2000), quant à elle, explique l'hypothèse de la maximisation de la richesse des managers de l'entreprise en plusieurs points dont l'aversion au risque des managers, le coût d'agence, l'habileté des managers etcetera.

\subsubsection{L'aversion au risque des managers}

Comme les actions procurent une fonction de profit linéaire, les managers actionnaires qui ont une aversion au risque (riscophobes), auront tendance à minimiser la volatilité de leurs profits, car ils ont une utilité 
anticipée de la richesse qui est significativement affectée par la variance des bénéfices anticipés de l'entreprise. Cela explique leur avantage à réduire le risque auquel ils sont exposés. Cliche (2000) déduit donc que plus un manager possède d'actions dans l'entreprise, plus ce dernier a une forte probabilité d'avoir des activités de couverture.

\subsubsection{Le coût d'agence}

Les managers ont peu d'intérêt à maximiser la valeur de la firme lorsqu'ils n'ont pas de droit de propriété dans la firme. Pour les motiver à maximiser leurs richesses, les actionnaires initient des contrats de compensation. Ces contrats lient le salaire du manager aux bénéfices de l'entreprise. Selon Cliche (2000), la convexité globale des contrats (contrats d'option sur action) amène les managers à prendre de plus grands risques parce que l'augmentation de la volatilité de la valeur de la firme fera augmenter la valeur de leurs options. Ces derniers n'ont donc pas intérêt à faire de la couverture. Les travaux de Tufano (1996), quant à lui, montrent que la couverture du risque du prix de l'or est motivée par l'aversion pour le risque des managers. Ses résultats attestent que les entreprises, dont les dirigeants disposent de stockoptions, couvrent moins le risque que celles dont les managers ont des actions de l'entreprise. Geczy et al. (1997) ont mené une étude sur l'utilisation des dérivés de change sur un échantillon de 372 entreprises du Fortune 500 en 1990. Cette étude confirme les résultats de la recherche de Tufano (1996). En ce qui les concerne, Smith et Stulz (1985) considèrent que les actionnaires ont intérêt à mettre au point des systèmes d'intéressement permettant d'aligner les objectifs des dirigeants sur les leurs. Pour cela, la rémunération des dirigeants doit comporter une partie fixe et une autre variable, en fonction de la performance de l'entreprise. Elle peut être une prime déterminée selon les résultats ou les options de souscription d'actions (stock-options). Ce type de rémunération fait en sorte que l'amélioration de la valeur de la société implique une augmentation de l'utilité espérée du dirigeant. Knopf et al. (2002), Rogers (2002) et Rajgopal et Shevlin (2002) proposent quant à eux d'utiliser à la fois l'effet de l'évolution du prix et de la volatilité de l'action sur la richesse du manager pour examiner la relation entre les préférences managériales et la couverture des risques. Car un manager acceptera de couvrir les risques financiers de l'entreprise que si la variation de sa richesse est très sensible au prix de l'action et se couvrira moins si celle-ci est davantage sensible à la volatilité du prix. Par conséquent, il est indispensable de prendre en considération l'effet du prix et de la volatilité quand il s'agit d'étudier l'effet de la détention de stock-options sur l'attitude du dirigeant envers 
le risque. L'augmentation de la volatilité entrainera une hausse de la valeur de l'option et, par conséquent, incite le dirigeant à chercher le risque. D'un autre côté l'augmentation du prix de l'action accroît la valeur de l'option mais également la probabilité de couverture.

\subsubsection{L'habileté des manager}

L'habilité des dirigeants de l'entreprise s'aperçoit au niveau des résultats financiers. D'autant plus que les personnes extérieures à l'entreprise ne possèdent que les informations financières, les managers ont une préférence pour la couverture des risques financiers qui apparaissent dans les données comptables contrairement aux autres risques. Ainsi le choix des instruments dérivés et celui de la politique de la gestion des risques ne se feront que s'ils améliorent les résultats de l'entreprise. A ce sujet, Cliche (2000) dira que la gestion des risques permet de réduire la quantité de "bruit" entourant les profits et augmente la quantité d'information disponible aux actionnaires. De ce fait, les actionnaires d'une firme, aux prises avec une grande asymétrie d'information au niveau des risques inobservables, bénéficient des avantages de la couverture. Ce point de vue, nous le pensons, s'appuie sur le modèle proposé par Breeden et Viswanathan (1998). Ces auteurs précisent que ce modèle ne repose pas sur la théorie du signal puisque l'activité de couverture n'est pas observée par les investisseurs externes. Pour eux, la couverture du risque est un moyen pour les dirigeants de révéler leurs compétences au marché.

L'habileté des dirigeants peut apparaître sous forme d'utilisation de pratique de couverture pour préserver leur emploi et/ou accroitre leur réputation. Amihud et Lev (1981) diront que le risque d'emploi est lié à une perte d'emploi, à une réputation entachée et un éventuel risque de révocation. Les dirigeants sont dans l'obligation de garantir la stabilité financière de l'entreprise et pour cela éviter tout évènement susceptible de nuire à l'entreprise et par ricochet mettre fin à leur contrat de travail. Demarzo et Duffie (1995) s'inscrivent dans la logique selon laquelle la performance de l'entreprise montre aux actionnaires la qualité du management du dirigeant. Leur modèle, publié en 1995, met en évidence le fait que la réputation est le principal souci des dirigeants. Dans ce modèle, le contenu informationnel du résultat de l'entreprise a deux effets :

- Effet sur la décision : l'information mise à la disposition des actionnaires détermine leur décision quant à savoir s'ils doivent continuer ou arrêter un projet d'investissement. 
- Effet sur la réputation: l'information révélée par les profits a un effet sur la réputation et cela découle les salaires futurs des dirigeants.

\subsection{L'effet de taille sur la décision d'adoption de couvertures de risques}

L'impact de la taille sur la décision d'adoption de couverture de risque fait sujet de débat dans la littérature. Plusieurs auteurs pensent que plus l'endettement et les coûts de faillites sont élevés, plus l'entreprise à un avantage à se couvrir. Pour Blazy (2000) le risque de faillite est plus élevé pour les Petites et Moyenne Entreprises (PME) qu'il ne l'est pas pour les grandes entreprises. Ce qui nous emmène à croire que les PME sont plus disposées à adopter les politiques de gestion de risque que les grandes entreprises. Et pourtant, ce sont les grandes entreprises qui ont suffisamment de moyens et d'expertise pour utiliser des politiques sophistiquées tels les produits dérivés. Elles ont les ressources financières qu'il faut pour embaucher le personnel avec les qualités qu'il faut pour l'utilisation des produits dérivés (Smith et Stulz, 1984; Block et Gallagher, 1986).

Dolde (1993) dira que les contraintes qui rendent l'utilisation des produits dérivés par les PME difficiles sont le manque de connaissance et d'accoutumance des gestionnaires des petites entreprises à ces actifs. Toutefois, le débat sur la relation entre la taille de l'entreprise et l'utilisation des produits dérivés demeure. Pendant que les études menées sur les déterminants de la gestion des risques par Nance et al. (1993), Mian (1996), Berkman et Bradbury (1996) et Geczy et al (1997) montrent une corrélation positive et significative entre la taille de l'entreprise et l'utilisation des produits dérivés, d'autres telles que celles menées par Tufano (1996), Gay et Nam (1998) et Haushalter (2000) établissent une corrélation négative.

\section{LA RELATION ENTRE GESTION DES RISQUES ET PERFORMANCE DES ENTREPRISES}

Les travaux antérieurs montrent un certain désaccord en ce qui concerne le lien entre les pratiques de gestion des risques et la performance financière des organisations (Assiènin et Ouattara, 2016). De nombreuses recherches (Hoyt et Liebenberg, 2011); Nocco et Stulz, 2006) établissent un lien positif entre la gestion des risques et 
la performance de l'entreprise. Pendant que d'autres comme celle de Pagach et Warr (2010), Ballantyne (2013), Razali et al. (2011), Ramlee et Ahmad (2015) soutiennent que l'adoption d'un système de gestion des risques n'a aucun effet sur la performance financière de l'entreprise.

\subsection{Aucun effet de la gestion des risques sur la performance}

Ramlee et Ahmad (2015) analysent dans leur étude les performances financières des entreprises non financières inscrites à la bourse de Bura sur la période 2009 à 2013. L'objectif principal de leur recherche était de savoir s'il existe une différence de comportement entre la mise en œuvre de la gestion des risques et la performance des entreprises avec et sans conseil d'administration. Les auteurs ont collecté les données sur un échantillon de 74 entreprises parmi lesquelles il y avait des entreprises avec un comité de gestion des risques et d'autres sans comité de gestion des risques. La performance financière (variable dépendante) a été mesurée par le ROE (Return on equity) ${ }^{2}$, le ROA (Return on asset) ${ }^{3}$ et le $\mathrm{Q}$ de Tobin. La variable indépendante utilisée est l'indice ERM (Enterprise Risk Management) qui est la version modifiée de Gordon et al. (2009). Les résultats de leur analyse des données de panel n'ont montré aucun impact significatif de la gestion des risques sur la performance des entreprises non financières en Malaisie. Selon cette étude, les entreprises appliquant la gestion des risques ne sont pas plus performantes que celles n'appliquant pas la gestion des risques.

Lukianchuk (2015) a, quant à lui, mené une étude sur l'impact de la gestion des risques sur la performance des petites et moyennes entreprises (PME). Les données utilisées ont été obtenues à partir d'une base de données financières fournissant les informations sur les entreprises basées au Royaume Unis et en Irlande du Nord. L'auteur a constitué un échantillon de 208 entreprises de tous les secteurs majeurs d'activité. Les variables indépendantes utilisées par l'auteur sont le montant des honoraires des auditeurs, le score de la qualité, la proportion d'hommes/femmes au conseil d'administration et la structure du conseil. Comme variable dépendante, il a utilisé le ROA et la volatilité des flux monétaires (VC). Les résultats de leur régression n'ont pas permis de vérifier le lien entre la gestion des risques et la performance.

Dans la même lancée, Pagach et Warr (2010) ont étudié les effets de la gestion des risques sur la performance des entreprises à partir de l'analyse des caractéristiques financières, des actifs et du marché. Ils 
ont utilisé un échantillon de 106 entreprises américaines qui ont embauché un gestionnaire des risques pour les accompagner dans la pratique de gestion des risques. Selon eux, les entreprises ne signalent pas publiquement leur adoption des pratiques de gestion des risques et ne donnent pas les détails de leur programme de gestion des risques. Ce qui les a conduits à se concentrer sur l'annonce de l'embauche d'agents de gestion des risques ou "risk managers" au sein des entreprises. Ils pensent en effet que l'embauche d'un "risk manager" devrait coïncider avec la décision d'adopter des pratiques de couverture. Les résultats, à partir de cet échantillon, ne leur ont pas permis de confirmer l'hypothèse selon laquelle la gestion des risques crée de la valeur. Pagach et Warr (2010) pensent que les partisans de Nocco et Stulz (2006), selon lesquels la gestion des risques impacte positivement la performance des entreprises, doivent fournir le programme de mise en ouvre du management des risques et les indicateurs à partir desquels les performances de ce programme peuvent être mesurées. Pour eux, des études supplémentaires sur la gestion des risques doivent être menées pour confirmer que la gestion des risques a des effets sur la performance des entreprises.

\subsection{Effet positif de la gestion des risques sur la performance}

Les recherches de Nocco et Stulz (2006) montrent comment la gestion des risques crée de la valeur pour les actionnaires. Ils présentent les avantages du dispositif de la gestion des risques. Selon eux, la gestion des risques crée de la valeur pour l'entreprise et elle est un avantage concurrentiel. Dans leur article, ils ne donnent pas des variables précises qui influencent la performance.

Pour ce qui concerne les recherches de Bertinetti, Cavezzali et Gardenal (2013), elles ont porté sur l'impact de l'adoption de la gestion des risques sur la valeur de l'entreprise et sur les déterminants du choix de la gestion des risques. Les auteurs ont travaillé sur un échantillon de 200 entreprises européennes de 2002 à 2011 qui appartiennent à l'indice STOXX Europe large, qui comportait à la fois des entreprises du secteur financier et non financier. En poursuivant un double objectif, ils ont essayé dans un premier temps de comprendre si la mise en œuvre de la politique de gestion des risques affectait la valeur de l'entreprise. Dans un deuxième temps, ils ont cherché à connaître les déterminants de l'adoption des pratiques de couverture. En utilisant une régression de panel à effets fixes pour le premier objectif et une analyse logistique à effets fixes pour le deuxième objectif, ils ont trouvé que le management des risques impactait positivement la valeur des 
entreprises européennes. Rappelons qu'ils ont utilisé le Q de Tobin comme variable dépendante et un ensemble de variable dépendante composé d'une variable de gestion des risques (présence d'un risk manager) et d'une autre de contrôle.

Kashif et Fong-Won (2015), quant à eux, proposent un cadre conceptuel pour enquêter sur l'impact de la gestion des risques sur la valeur de l'entreprise à travers la valeur économique ajoutée (EVA). L'étude adopte un cadre de mise en ouvre de la gestion des risques qui comporte trois dimensions à savoir: la structure, la gouvernance et le processus. Ils concluent qu'il y a un effet positif de la gestion des risques sur la performance des entreprises.

Graham et Roger (2002) ont porté leur étude sur l'utilisation des produits dérivés pour couvrir les risques, le taux d'échange et le taux d'intérêt. Ils ont constitué un échantillon de 442 entreprises et ont utilisé une régression multivariée avec pour variables indépendantes la convexité de l'impôt (avantage fiscal résultant d'une réduction de la volatilité), le ratio dette sur actif, le ratio du marché boursier, le ratio recherche et développement sur actifs, les dépenses en immobilisation par rapport aux actifs, le rendement en dividende, le ratio des ventes à l'étranger sur ventes totales et le ROA avant impôts. Les variables dépendantes sont le ratio d'endettement et les produits dérivés. Les résultats de cette recherche montrent une corrélation positive entre la valeur de l'action et l'utilisation des produits dérivés pour la gestion des risques du taux d'intérêt et du taux de change. L'étude montre aussi une forte corrélation entre la valeur de l'action et l'utilisation de dérivés des prix des marchandises. Ainsi, ils parviennent à la conclusion que les entreprises se couvrent pour augmenter leur capacité d'endettement et les avantages fiscaux résultant de la couverture et ajoutent environ $1,1 \%$ à la valeur de l'entreprise. La gestion des risques financiers influence positivement la valeur de l'entreprise.

\subsection{Résultats mitigés}

L'étude de Assiènin et Ouattara (2016) avait pour objectif d'analyser l'impact de la gestion des risques opérationnels sur la performance des entreprises non financières en Côte d'Ivoire. Sur un échantillon de 70 entreprises non financières avec un taux de réponse de $63 \%$, ils ont collecté les informations sur la gestion des risques de ces entreprises à partir d'un questionnaire et complété ces informations par des données issues de la banque des données financières ivoirienne. La performance financière a été mesurée par ROE et l'EBE (Excédent brut 
d'exploitation). Les analyses menées sur les données recueillies à partir d'une régression d'un modèle linéaire conduisent aux résultats suivants: la culture du risque et les dotations aux amortissements et provisions ont un impact positif sur le ROE et l'EBE, tandis que les réserves et la protection contractuelle les impactent négativement. Nous pensons que les résultats mitigés de cette étude pourraient être à l'origine de ce débat concernant l'influence des pratiques de couvertures sur la performance.

C'est sans doute ce qui a inspiré Florio et Leoni (2017) qui ont étudié la relation entre l'étendue de la mise en ouvre des systèmes de gestion des risques de l'entreprise et la performance des entreprises côtés en Italie. La population étudiée était de 416 entreprises non financières cotées à la bourse de Milan. Les données recueillies concernaient la période de 2011 à 2013. Les résultats, issus d'analyse de régressions de moindres carrés ordinaires, montrent que les entreprises ayant des niveaux élevés de mise en œuvre de la gestion des risques d'entreprise présentent des performances supérieures, à la fois en termes de performance financière (ROA) et d'évaluation du marché ( $Q$ de Tobin). La gestion des risques a été appréciée par la présence d'un risk manager, d'un comité de gestion des risques et la fréquence du reporting entre le comité de risque et le conseil d'administration. On déduit à partir de cette étude que pour qu'il y ait impact de la performance, il faut un niveau élevé de mise en ouvre de la gestion des risques.

\section{CONCLUSION}

Notre analyse de la littérature nous a permis de parcourir les théories de la firme qui, selon nous, constituent le socle du management du risque. Par ailleurs, elle nous a permis d'identifier les déterminants théoriques de la couverture du risque et d'apprécier l'impact des pratiques de gestion des risques sur la performance des entreprises.

Toutefois, nous pensons que des études supplémentaires sur les effets de l'adoption des pratiques de gestion sur la performance globale des entreprises seront les bienvenues car elles permettront de déterminer d'autres indicateurs de performance (surtout les indicateurs non financiers) qui sont impactés par la mise en ouvre d'un programme de management des risques. Aussi, nous proposons comme prolongement de ce travail une étude sur l'évolution les théories qui constituent les fondements de la gestion des risques. L'objectif de ce travail pourrait 
être, dans cette perspective, de savoir si les théories qui justifiaient l'adoption des politiques de gestion des risques sont les mêmes aujourd'hui et de déterminer les nouveaux fondements de la gestion des risques qui guideraient les gestionnaires des risques.

\section{BIBLIOGRAPHIE}

[1] Abecassis, C. (1997). Les coûts de transaction: état de la théorie. Réseaux, 15 (84), 9-19.

[2] Alchian, A. A. and Demsetz, H. (1972). Production, information costs, and economic. The American review, 62 (5), 777-795.

[3] Amihud, Y. et Lev, B. (1981). Risk reduction as a managerial motive for merger. Bell Journal of Economics, 12, 605-617.

[4] Aretz, K., Bartram, S. M. et Dufey G. (2007). Pourquoi se couvrir? Rations pour la couverture de l'entreprise et les implications de la valeur. The journal of risk finance, 8 (5), 434-449.

[5] Assiènin, A. et Ouattara, A. (2016). L'impact de la gestion des risques opérationnels sur la performance des entreprises non financières. Finance E Finance Internationale. DOI : 10.12816/0040364

[6] Ballantyne, F.(2013). Évaluation du modèle pour déterminer si des transformations logarithmiques sont nécessaires dans l'allométrie: un commentaire sur l'échange entre Packard (2009) et Kerkhoff \& Enquist (2009). The Journal of Theoretical Biology, 31,7 418-421.

[7] Béjean, S., Midy, F. et Peyron, C. (1999). La rationalité simonienne: interprétations Journal of Financial Economics et enjeux épistémologiques. Document de travail, LATEC, Laboratoire d'Analyse et des Techniques EConomiques, Université de Bourgogne, France. Repéré à https://ideas. repec.org/p/lat/lateco/1999-14.html

[8] Berkman, H. et Bradbury, M.E. (1996). Empirical evidence on the corporate use of derivatives, Financial Management, 26, 5-13.

[9] Bertinetti, G. S., Cavezzali, E. et Gardenal, G. (2013). The effect of the enterprise risk management implementation on the firm value of European companies. Working Paper n. 10/2013 of the Department of Management at Università Ca' Foscari Venezia, Italie.

[10] Bessembinder, H. (1991). Forward Contracts and firm value: Investment incentive and contracting effects. Journal of Financial and Quantitative Analysis, 26, 519-532. 
[11] Blauner, R. (1964). Alienation, freedom and technology. Phoenix Books.

[12] Blazy, R. (2000). La faillite: Eléments d'analyse économique. Paris: Economica.

[13] Block, S. B. et Gallagher, T. J. (1986). Une étude empirique de l'utilisation des futures et des options par gestion corporative. Revue financière, 21(8). DOI : 10.1111 / j.1540-6288. 1986.tb00672.x

[14] Bordes, C. (1999). Droit, économie et justice dans le secteur bancaire. Banque et risque systémique (version préliminaire). Document de travail, Université Paris1, France). https://www.courdecassation.fr/IMG/File/ risque_systemique_bordes.pdf

[15] Brealey, R. A. et Myers, S. C. (1984). Principles of Corporate Finance, McGraw-Hill, 1981. Cette première édition a fait l'objet d'une traduction en français et d'une adaptation au contexte canadien: Brealey, R. A, Myers, S. C. et Charette, F. (1984). Principes de gestion financière des sociétés, McGraw-Hill.

[16] Breeden, D. et Viswanathan, S. (1998). Why do firms hedge? An asymmetric information model. Working paper, Duke University. https://static. secure.website/wscfus/8149792/uploads/Breeden_1991_Viswanathan_ Why_Do_Firms_Hedge_Unpublished.pdf

[17] Burns, T. et Stalker, G. M. (1961). The management of innovation. London: Tavistocks Publications.

[18] Chanut O., Paché, G. et Wagenhausen, F. (2012). Logistique urbaine: refonder les logiques d'intermédiation, Management $\&$ Avenir,51 (1), 186207. DOI : $10.3917 /$ mav.051.0186

[19] Charreaux G. et Pitol-Belin J. P. (1992). Les théories des organisations. Dans Simon, Y. et Joffre, P. (1997). Encyclopédie de gestion. Paris, France: Economica.

[20] Cliche J.-A. (2000). Les déterminants de la gestion des risques par les entreprises non financières: une revue de la littérature (Mémoire de mâ̂trise, Ecole des hautes études commerciales (HEC), Montréal, Canada). Repéré à: http://chairegestiondesrisques.hec.ca/wp-content/uploads/pdf/ cahiers-recherche/00-02.pdf

[21] Coase, R. H. (1937). The nature of the firm. Economica, 4, 386-405.

[22] Cozic, M. (2018). La rationalité limitée. Dans Walliser, B. (dir) Economie Cognitive (p. 199-222), MSH/Ophrys. 
[23] Dean, U. et Jay, B. (1984). Perspectives in Organizations: Resource Dependence, Efficiency and Population. Academy of Management Review, 9 (3), 471-481.

[24] Demarzo, P. et Duffie, D. (1995). Corporate Incentives for hedging and hedge Accounting. The Review of Financial Studies, 8(3), 743-771.

[25] Demsetz, H. (1967). Towards a Theory of Property Rights. American Economic Review, 57 (2), 347-359.

[26] Desbrières, P. (1990). Participation financière des salariés et organisation interne de l'entreprise. Revue d'économie industrielle, 54 (4), 44-67.

[27] Dolde, W. (1993) The trajectory of corporate financial risk management, Journal of Applied Corporate Finance, 6 (3), 33-41.

[28] Emery, F. E. et Trist, G. L. (1969). Socio-technical systems Dans Emery (ed.), Systems Thinking. Harmondsworth, England: Penguin Books.

[29] Fayol, H. (1962). Administration industrielle et générale. Paris: Dunod.

[30] Florio, C. et Leoni, G. (2017). Enterprise risk management and firm performance: the Italian case, the British Accounting Review, 49 (1), 56-74.

[31] Friedman, M. (1953). The methodology of positive economics. Dans Essays in positive economics, Chicago: The University of Chicago Press.

[32] Froot, K. A., Scharfstein, D. S. et Stein, J. C. (1993). Risk management: coordinating Corporate investment and financing Policies. The journal of finance, 48 (5), 1629-1658.

[33] Furubotn, E) et Pejovich, S. (1972). Property Rights and Economy Theory: A Survey of Recent Literature. Journal of Economic Literature, 10 (4), 1137-1162.

[34] Furubotn, E. et Pejovich, S. (1972). Property Right, Economic Decentralisation and the Evolution of the Yugoslav Firm. Journal of Law and Economics, 16 (2), 275-302.

[35] Gay, G. D. et Nam, J. (1998). The underinvestment problem and corporate derivatives use. Financial management, 27 (4), 53-69.

[36] Geczy, C., Minton, B. A. et Schrand, C. (1997). Why firms use currency derivatives Journal of Finance, 52 (4), 1323-1354.

[37] Gordon, L. A., Loeb, M. P. and Tseng, C. H. (2009). Enterprise Risk Management and Firm Performance: A Contingency Perspective. Journal of Accountant and Public Policy, 28 (4), 301-327.

[38] Graham, J. R. et Smith, C. W. (1999). Tax incentives to hedge. Journal of Finance, 54 (6), 2241-2261. 
[39] Graham, J. R. et Rogers, D. A. (2002). Do firms hedge in response to tax incentives. Journal of Finance, 57 (2), 815-839.

[40] Hannan, M. T., et Freeman, J. (1977). The population Ecology of Organizations. American Journal of Sociology, 82 (5), 929-964.

[41] Hatch, M. J. (2000). Théorie des organisations. De l'intérêt de perspectives multiples, Bruxelles: De Boeck université.

[42] Haushalter, G. D. (2000). Financing Policy, Basis Risk, and Corporate Hedging: Evidence from Oil and Gas Producers. Journal of Finance, 55 (1), 107-152.

[43] Herzberg, F., Mausner, B. et Snyderman, B. (1959). The motivation to work. Oxford, England: John Wiley.

[44] Hoyt, R. E. et Liebenberg, A. P. (2011). The Value of Enterprise Risk Management: Evidence from the U.S. Insurance Industry. Journal of risk and insurance, 78 (4), 795-822.

[45] Jensen, M. C. et Meckling, W. H. (1976). Theory of the firm: managerial behavior, agency costs and ownership structure. Journal of Financial Economics, 3 (4), 305-360.

[46] Jost, S. (2004) La théorie des coûts de transaction de Williamson et la surveillance des banques dans l'UE (Mémoire de maîtrise, Institut européen de l'Université de Genève, Suisse). Repéré à https://archiveouverte.unige.ch/unige: 20310

[47] Karim, B. K. et Souad, L. J. (2010). Les déterminants de la couverture des risques en France. La revue des sciences de gestion, 245-246 (5-6), 119-127.

[48] Kashif, M. S. et Fong-Won, L. (2015). A Conceptual Framework for Enterprise Risk Management performance measure through Economic Value Added. Global Business and Management Research: An International Journal, 7 (2).

[49] Knopf, J., Nam, J. et Thornton J. J. (2002). The volatility and price sensitivities of managerial stock option portfolios and corporate hedging. Journal of Finance, 57 (2), 801-813.

[50] Laville, F. (1998). Modélisations de la rationalité limitée: de quels outils dispose-t-on? Revue Economique, 49 (2), 335-365.

[51] Lawrence, P. R. et Lorsch, J. W. (1973). Adapter les structures de l'entreprise. Paris, France: Les éditions d'organisation. 
[52] Lukianchuk, G. (2015). The impact of enterprise risk management on firm performance of small and medium enterprises. European Scientific Journal, 11 (13), 408-417.

[53] Mayer, D. and Smith, C. (1982). On the corporate demand for Insurance. Journal of business, 55 (2), 281-296.

[54] Mayo, E. (1949). The buman problems of an industrial civilization. London: Routledge.

[55] Maslow, A. H. (1954). Motivation and personality. New York: Harper \& Row.

[56] Mefteh, S. (2005). Les déterminants de la gestion des risques financiers des entreprises non financières: une synthèse de la littérature. Cahier de recherche n`2 005-03. Université Paris Dauphine, France.

[57] Mellios, C. (2003). La gestion des risques financiers par les entreprises: explications théoriques versus études empiriques. Revue d'économie financière, 72, 243-264.

[58] Mian, S. L. (1996). Evidence on the Corporate Hedging Policy. Journal of Financial and Quantitative Analysis, 31 (3),419-439.

[59] Mintzberg, H., Ahlstrand, B. et Lampel, J. (2005). Safari en pays stratégie - L'exploration des grands courants de la pensée stratégique. Pearson Education France, Village Mondial.

[60] Modigliani, F. et Miller, M. (1958). The cost of capital, corporation finance, and the theory of investment. American Economics Review, 48 (3), 261-297.

[61] Nance, D., Smith, C. et Smithson, C. W, (1993). On the determinants of corporate Hedging. Journal of Finance, 48 (1), 267-284.

[62] Nocco, B. W. et Stulz, R. M. (2006). Enterprise Risk Management: Theory and Practice. Journal of Applied Corporate Finance, 18(4), 8-20. Available at SSRN : http://ssrn.com/abstract=963398

[63] Orlean, A. (1994). Analyse économique des conventions. Paris: Presses Universitaires de France.

[64] Pagach, D. P. and Warr, R. S. (2010). The effects of enterprise risk management on firm performance. Available at http://dx.doi.org/10.2139/ ssrn. 1155218

[65] Pejovich, S., Djurdjevac, V. et Furubotn, E. G. (1978). The Economies of Property Rights. Revue d'études comparatives Est-Ouest, 9 (1), 223-226.

[66] Pfeffer, J. et Salancik, G. R. (1978). The External Control of Organizations - A Resource Dependence Perspective. New York: Harper \& Row. 
[67] Ponssard, J. P. (1994). Formalisation des connaissances, apprentissage organisationnel et rationalité interactive. Dans Orlean, A. Analyse économique des conventions (p. 169-198). Paris, France: Presses Universitaires de France.

[68] Popper, K. R. (1963). Conjectures et réfutations. Traduction française de Launais et Launais. Paris, France: Payot.

[69] Raïs, H. M. (2012). Gestion des risques: mesures et stratégies: analyse empirique de la gestion des risques dans les entreprises non financières françaises (Thèse de doctorat en Sciences de gestion, Université Toulouse Capitale 1, Toulouse, France). Repéré à www.theses.fr/2012TOU10063

[70] Rajgopal, S. et Shevlin, T. (2002). Empirical evidence on the relation between stock option compensation and risk taking. Journal of Accounting and Economics, 33 (2), 145-171.

[71] Ramlee, R. et Ahmad, N. (2015). Panel Data Analysis on the Effect of Establishing the Enterprise Risk Management on Firms' Performances Proceedings of 4th European Business Research Conference, Imperial College, London, UK, ISBN : 978-1-922069-72-6

[72] Razali, A. R., Yazid, A. S., et Tahir, I. M. (2011). The Determinants of Enterprise Risk Management (ERM) Practices in Malaysian Public Listed Companies. Journal of Social and Development Sciences, 1(5), 202-207.

[73] Robichek, A. A., and Myers, S. C. (1966). Conceptual Problems in the Use of Risk-Adjusted Discount Rates. Journal of Finance, 21 (4), 727-730.

[74] Rogers, D. A. (2002). Does executive portfolio structure affect risk management? CEO risk-taking incentives and corporate derivatives usage. Journal of Banking and Finance, 26, 271-295.

[75] Simon, H. (1955). A behavioral model of rational choice. Quarterly Journal of Economics, 69 (2), 99-118.

[76] Simon, H. (1978). Rationality as Process and as Product of Thought. The American Economic Review, 68, (2), 1-16.

[77] Simon, Y. et du Montcel H. T. (1977). Théorie de la firme et réforme de l'entreprise. Revue économique, 28 (3), 321-351.

[78] Smith, C. et Stulz, R. (1984). Optimal Hedging Policies. Journal of Financial and Quantitative Analysis, 19 (2), 127-139.

[79] Smith, C. and Stulz, R. (1985). The determinants of firms' Hedging Policies. Journal of Financial and Quantitative Analysis, 20 (4), 391-405. 
[80] Smith, C. W. et Watts, R. L. (1992). The investment opportunity set and corporate Financing dividend and compensation policies. Journal of financial economics, 32 (3), 263-292.

[81] Stulz, R. (1996). Rethinking Risk management. Journal of Applied Corporate Finance, 9 (3), 8-24.

[82] Taylor, F. (1929). The principals of scientific management. New York and London: Harper.

[83] Tinel, B. (2004). Que reste-t-il de la contribution d'Alchian et Demsetz à la théorie de l'entreprise? Revue cabiers d'économie Politique, 46 (1), 67-89.

[84] Tufano, P. (1996). Who manages risk? An empirical examination of risk management practices in the gold mining industry. Journal of Finance, 9 (1), 1097-1137.

[85] Vernimmen, P. (2012). Finance d'entreprise. France: DALLOZ.

[86] Viviani, J. L. (1994). Ambigü̈té. Cahier de recherche CRIEGE, université de Paris-XIII, nº 4 103Walliser, B. (1989). Instrumental rationality and cognitive rationality. Theory and Decision, 27 (1-2), 7-36.

[87] Williamson, O. (1975). Market and Hierarchies: Analysis and Antitrust Implications New York: the Free Press.

[88] Williamson, O. (1979). Transaction Costs Economics: The Governance of Contractual Relations, Journal of Law and Economics, 22 (2), 233-261.

[89] Williamson, O. E. (1985). The Economic Institutions of Capitalism. New York: The Free Press.

[90] Williamson, O. (1994). Les Institutions de l'Economie. Traduit par R. Coeurderoy et E. Maincent sous la direction de M. Ghertman, de: The Economic Institutions of Capitalism (1985). Paris, France: InterEditions.

\section{NOTES}

1 Doctorant à I'Université Félix Houphouët Boigny de Cocody, Côte d'Ivoire, BP 1524 Abidjan 21/ (+225) 777772 43/assimelo@gmail.com.

2 ROE: Rentabilité des capitaux propres en français.

3 ROA: Rentabilité des actifs en français. 